Revista Mexicana de Astronomía y Astrofísica, 55, 289-303 (2019)

DOI: https://doi.org/10.22201/ia.01851101p.2019.55.02.15

\title{
MILLIMETER AND FAR-IR STUDY OF THE IRDC SDC 341.232-0.268
}

\author{
M. M. Vazzano ${ }^{1}$, C. E. Cappa ${ }^{2}$, V. Firpo ${ }^{4,5}$, C. H. López-Caraballo ${ }^{6}$, M. Rubio ${ }^{3}$, and N. U. Duronea ${ }^{1}$
}

Received April 1 2019; accepted July 172019

\begin{abstract}
We analyze the molecular gas and dust associated with the infrared dark cloud SDC 341.232-0.268 in order to investigate the characteristics and parameters of the gas, determine the evolutionary status of four embedded EGO candidates, and establish possible infall or outflow gas motions. We base our study on ${ }^{12} \mathrm{CO}(2-1)$, ${ }^{13} \mathrm{CO}(2-1)$, and $\mathrm{C}^{18} \mathrm{O}(2-1)$ data obtained with the APEX telescope, molecular data of high density tracers from the MALT90 survey and IR images from Spitzer, Herschel and ATLASGAL. The study reveals two clumps at $-44 \mathrm{~km} \mathrm{~s}^{-1}$ towards the IRDC, with densities of $>10^{4} \mathrm{~cm}^{-3}$, typical of IRDCs, while high density tracers show $\mathrm{H}_{2}$ densities $>10^{5}$. FIR images reveals the presence of cold dust linked to the molecular clumps and EGOs. A comparison of the spectra of the optically thin and optically thick molecular lines towards the EGOs suggests the existence of infall and outflow motions.
\end{abstract}

\section{RESUMEN}

Analizamos el gas molecular y el polvo asociado a la nube oscura infrarroja SDC 341.232-0.268 con el fin de investigar las características y parámetros físicos del gas, determinar el estado evolutivo de los cuatro EGOs embebidos y establecer posibles movimientos de acreción o flujo molecular. Nos basamos en datos de ${ }^{12} \mathrm{CO}(2-1),{ }^{13} \mathrm{CO}(2-1)$ y $\mathrm{C}^{18} \mathrm{O}(2-1)$ obtenidos con el telescopio APEX, trazadores de alta densidad extraídos de MALT90, e imágenes infrarrojas de Spitzer, Herschel y ATLASGAL. El estudio revela dos grumos moleculares a $-44 \mathrm{~km} \mathrm{~s}^{-1}$ coincidentes con la IRDC con una densidad $>10^{4} \mathrm{~cm}^{-3}$, típica de IRDCs. Los trazadores de alta densidad arrojan densidades de $\mathrm{H}_{2}>10^{5}$. Las imágenes en el lejano IR muestran polvo frío asociado a los grumos moleculares y a los EGOs. La comparación de espectros moleculares ópticamente gruesos y finos sugiere la existencia de acreción y flujos moleculares.

Key Words: dust, extinction - ISM: clouds - ISM: individual objects: IRDC SDC 341.232-0.268 - ISM: kinematics and dynamics - ISM: molecules - stars: formation

\section{INTRODUCTION}

Infrared dark clouds (IRDCs) are sites of recent star formation within molecular clouds. These are detected as dark silhouettes against a bright back-

\footnotetext{
${ }^{1}$ Instituto Argentino de Radioastronomía, CONICET, CCT-La Plata, Villa Elisa, Argentina.

${ }^{2}$ Facultad de Ciencias Astronómicas y Geofísicas, Universidad Nacional de La Plata, La Plata, Argentina.

${ }^{3}$ Departamento de Astronomía, Universidad de Chile, Chile.

${ }^{4}$ Gemini Observatory, Southern Operations Center, La Serena, Chile.

${ }^{5}$ Departamento de Astronomía y Física, Universidad de la Serena, La Serena, Chile.

${ }^{6}$ Departamento de Matemáticas, Universidad de La Serena, La Serena, Chile.
}

ground in the mid-infrared. These regions, which are also detected in the FIR, are believed to be places that may contain compact cores which probably host the early stages of high-mass star formation (e.g., Rathborne et al. 2007; Chambers et al. 2009). In fact, their cold $(\mathrm{T}<25 \mathrm{~K})$ and dense $\left(10^{2}-10^{4} \mathrm{~cm}^{-3}\right)$ ambient conditions, and their sizes of $\approx 1-3 \mathrm{pc}$ and masses of $10^{2}-10^{4} M_{\odot}$ (Rathborne et al. 2006, 2007) favor star formation mechanisms. Evidences of active high-mass star formation in IRDCs are inferred from the presence of ultracompact HiI regions (Battersby et al. 2010), hot cores (Rathborne et al. 2008), embedded $24 \mu \mathrm{m}$ sources (Chambers et al. 2009), maser emission (Wang et al. 2008; Chambers et al. 


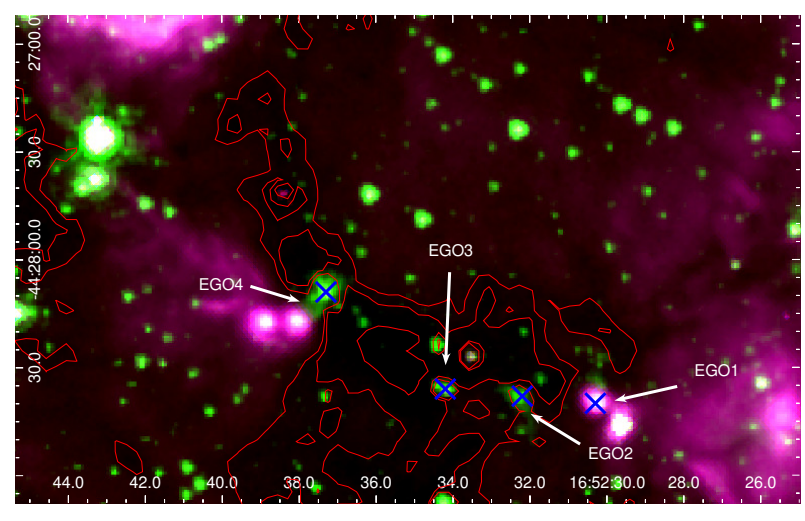

Fig. 1. Composite image showing the Spitzer-IRAC emission at 4.5 (green) and $8.0 \mu \mathrm{m}$ (red), and Spitzer-MIPS emission at $24 \mu \mathrm{m}$ (blue) of the region of SDC 341.232-0.268. Contours correspond to the emission at $8.0 \mu \mathrm{m}$ at 80,90 , and $95 \mathrm{MJy} / \mathrm{ster}$. The positions of the candidate EGOs are marked with blue crosses. The color figure can be viewed online.

2009), and/or outflow and infall processes (e.g. Sanhueza et al. 2010; Chen et al. 2011; Ren et al. 2011; Beuther et al. 2002; Zhang et al. 2007).

Many IRDCs are also associated with extended enhanced $4.5 \mu \mathrm{m}$ emission (named extended green objects, EGOs, for the common coding of the [4.5] band in green in the 3-color composite IRAC images), the so-called "green fuzzies", indicating the presence of shocks. Following photometric criteria, Cyganowski et al. (2008) suggested that most of EGOs fall in the region of the color-color space occupied by the youngest MYSOs, and are surrounded by accreting envelopes (see Figure 13 in their work). This hypothesis is supported by Chen et al. (2010), whose observations are consistent with the speculation that EGOs trace a population with ongoing outflow activity and an active rapid accretion stage of massive protostellar evolution. The emission at $4.5 \mu \mathrm{m}$ includes both $\mathrm{H}_{2}(\mathrm{v}=0-0, \mathrm{~S}(9,10,11))$ lines and $\mathrm{CO}(\mathrm{v}=1-0)$ band heads (Cyganowski et al. 2008), which are excited by shocks, such as those expected when protostellar outflows impinge on the ambient interstellar medium (ISM) (Cyganowski et al. 2008).

Using IRAC images, Peretto \& Fuller (2009) catalogued and characterized many IRDCs using 8 and $24 \mu \mathrm{m}$ Spitzer data. In this catalogue, the IRDCs were defined as connected structures with $\mathrm{H}_{2}$ column density peaks $N_{\mathrm{H}_{2}}>2 \times 10^{22} \mathrm{~cm}^{-2}$ and boundaries defined by $N_{\mathrm{H}_{2}}=1 \times 10^{22} \mathrm{~cm}^{-2}$. Single-peaked structures found in the $\mathrm{H}_{2}$ column density maps were identified by the authors as fragments. These dark and dense patches are associated with molecular gas and dust emission (Sanhueza et al. 2012).

As a part of a project aimed at studying in detail the physical properties of the IR bubble complex S21-S24, we present here an analysis of the molecular gas and dust associated with SDC 341.232-0.268, a poorly studied IRDC of the southern hemisphere, which is member of this complex. Our aims are to determine the physical parameters and kinematics of the gas and to characterize the interstellar dust associated with the IRDC, to investigate the presence of embedded young stellar objects, and to establish possible infall or outflow gas motions. To accomplish this project, we analyze molecular data of the ${ }^{12} \mathrm{CO}(2-1),{ }^{13} \mathrm{CO}(2-1)$, and $\mathrm{C}^{18} \mathrm{O}(2-1)$ lines obtained with the APEX telescope, and $\mathrm{HCO}^{+}(1-0), \mathrm{HNC}(1-0)$, and $\mathrm{N}_{2} \mathrm{H}^{+}(1-0)$ lines from the MALT90 survey (Jackson et al. 2013; Foster et al. 2013, 2011) ${ }^{7}$, mid- and far-IR continuum data from Spitzer-IRAC, Herschel-PACS and -SPIRE, and ATLASGAL (APEX) images (Schuller et al. 2009). We introduce the IRDC SDC 341.232-0.268 in $\S 2$, the used database in $\S 3$, the results of the molecular gas in $\S 4$, and those of the infrared emission in $\S 5$; signs of star formation are discussed in $\S 6$, and a summary in $\S 7$.

\section{IRDC SDC 341.232-0.268}

The IRDC SDC 341.232-0.268 (RA, Dec. $(\mathrm{J} 2000)=16: 52: 35.67,-44: 28: 21.8)$ has a mid-IR radiation field intensity $I_{\mathrm{MIR}} \approx 88.3 \mathrm{MJy}$ ster $^{-1}$ at $8.0 \mu \mathrm{m}$ and a peak opacity of 1.09. From the Multiband Infrared Photometer for Spitzer (MIPS) images Peretto \& Fuller (2009) detected a $24 \mu \mathrm{m}$ point source in the field of the IRDC, and two clumps. The clumps were classified by Bergin \& Tafalla (2007) as structures with sizes $\approx 10^{-1}-100 \mathrm{pc}$, masses $\approx 10-10^{3} M_{\odot}$, and volume densities $\approx 10^{3}-10^{4} \mathrm{~cm}^{-3}$.

Figure 1 shows the Spitzer-IRAC images at 4.5 (green) and $8.0 \mu \mathrm{m}$ (red), and the Spitzer-MIPS image at $24 \mu \mathrm{m}$ (blue) in the region of SDC 341.232-0.268. Green contours correspond to low emission levels at $8 \mu \mathrm{m}$, revealing a region of high extinction at $8.0 \mu \mathrm{m}$ at the location of the IRDC, in contrast with the environment, a typical feature of these objects. The $90 \mathrm{MJy} / \mathrm{ster}$ contour delineates the IRDC. Four candidate EGOs catalogued by Cyganowski et al. (2008) appear projected toward this region: the "likely" MYSO outflow candidates G 341.22-0.26(b)(R.A., Decl.(J2000) = 16:52:30.3,

\footnotetext{
${ }^{7}$ http://malt90.bu.edu/data.html
} 
-44:28:40.0), G 341.22-0.26(a) (16:52:32.2, $-44: 28: 38.0)$, the "possible" MYSO outflow candidate G 341.23-0.27 (16:52:34.2, $-44: 28: 36.0)$, and the "likely" MYSO outflow candidate G 341.24-0.27 (16:52:37.3, -44:28:09.0). These authors categorized the EGOs as a "likely" or "possible" MYSO outflow candidate based primarily on the angular extent of the extended excess $4.5 \mu \mathrm{m}$ emission. Any source in which it was possible to confuse multiple nearby point sources and/or image artifacts from a bright IRAC with truly extended $4.5 \mu \mathrm{m}$ emission was considered a "possible" candidate; these are likely still good YSO candidates, but not necessarily MYSOs with outflows, and so likely to be actively accreting.

From here on, they will be named EGO 1, EGO 2, EGO 3, and EGO 4, in increasing order of R.A. EGO 1 is associated with 8 and $24 \mu \mathrm{m}$ emission and is located in the border of the IRDC, while EGOs 2, 3, and 4 are detected at 4.5 and $8 \mu \mathrm{m}$. EGO 1 and EGO 4 coincide with ATLASGAL Compact Sources AGAL 341.219-00.259 and AGAL 341.236-00.271. Their integrated flux densities are $12.85 \pm 2.20 \mathrm{Jy}$ and $16.81 \pm 2.80 \mathrm{Jy}$, and their effective radii are $\approx 37^{\prime \prime}$ and $47^{\prime \prime}$, respectively (Contreras et al. 2013).

Methanol maser emission was detected towards EGO 2 and EGO 4 at 6 and $95 \mathrm{GHz}$, within the velocity range -43 to $-52 \mathrm{~km} \mathrm{~s}^{-1}$ (Caswell et al. 2010; Chen et al. 2011; Hou \& Han 2014; Yang et al. 2017). Methanol masers provide a signpost to the very earliest stages of the massive star formation process, prior to the onset of the UCHII region phase. They are associated with embedded sources whose bolometric luminosities suggest that they will soon become OB stars (Burton et al. 2002; Sobolev et al. 2005). The methanol masers are independent tracers and they give proven signatures of ongoing star formation (see Ellingsen 2006; Breen et al. 2013). Masers of water or hydroxyl have also been detected in in star-forming regions, as well as in evolved stars or supernova remnants. Therefore, the presence of these maser types in the vicinity of these EGOs could be a signspot of ongoing star formation.

\section{DATABASE}

\subsection{Molecular Line Observations}

The ${ }^{12} \mathrm{CO}(2-1),{ }^{13} \mathrm{CO}(2-1)$, and $\mathrm{C}^{18} \mathrm{O}(2-1)$ data were acquired with the APEX-1 receiver of the Swedish Heterodyne Facility Instrument (SHeFI; Vassilev et al. 2008) in the Atacama Pathfinder EXperiment (APEX) telescope, located in the Puna de
TABLE 1

OBSERVATIONAL PARAMETERS OF THE MOLECULAR TRANSITIONS

\begin{tabular}{lcccc}
\hline Transition & $\begin{array}{c}\nu_{0} \\
\mathrm{GHz}\end{array}$ & $\begin{array}{c}\theta \\
\prime\end{array}$ & $\begin{array}{c}\Delta v_{\text {res }} \\
\mathrm{km} \mathrm{s}^{-1}\end{array}$ & $\begin{array}{c}\mathrm{rms} \\
\mathrm{K}\end{array}$ \\
\hline${ }^{12} \mathrm{CO}(2-1)$ & 230.538 & 30 & 0.1 & 0.35 \\
${ }^{13} \mathrm{CO}(2-1)$ & 220.398 & 28.5 & 0.1 & 0.35 \\
$\mathrm{C}^{18} \mathrm{O}(2-1)$ & 219.560 & 28.3 & 0.1 & 0.35 \\
$\mathrm{HNC}^{2}(1-0)$ & 90.664 & 38.0 & 0.11 & 0.35 \\
$\mathrm{HCO}^{+}(1-0)$ & 89.189 & 38.0 & 0.11 & 0.35 \\
$\mathrm{~N}_{2} \mathrm{H}^{+}(1-0)$ & 93.173 & 38.0 & 0.11 & 0.35 \\
\hline
\end{tabular}

Atacama (Chile). The backend for the observations was the eXtended bandwidth Fast Fourier Transform Spectrometer2 (XFFTS2) with a $2.5 \mathrm{GHz}$ bandwidth divided into 32768 channels. The main parameters of the molecular transitions (rest frequency $\nu_{0}$, halfpower beam-width $\theta$, velocity resolution $\Delta \mathrm{v}_{\text {res }}$, and rms noise of the individual spectra obtained using the OTF mode) are listed in Table 1. The selected off-source position free of molecular emission was RA, Dec. $(J 2000)=(16: 36: 40.56,-42: 03: 40.6)$.

Calibration was done using the chopper-wheel technique. The antenna temperature scale was converted to the main-beam brightness-temperature scale by $T_{\mathrm{mb}}=T_{\mathrm{A}} / \eta_{\mathrm{mb}}$, where $\eta_{\mathrm{mb}}$ is the main beam efficiency. For the SHeFI/APEX-1 receiver we adopted $\eta_{\mathrm{mb}}=0.75$. Ambient conditions were good, with a precipitable water vapor (PWV) between $1.5-2.0 \mathrm{~mm}$.

The molecular spectra were reduced using the CLASS90 software of the IRAM's GILDAS software package.

In addition, we used molecular data from the Millimetre Astronomy Legacy Team 90 GHz Survey (MALT90) taken with the Mopra spectrometer (MOPS). We used beam efficiencies between 0.49 at $86 \mathrm{GHz}$ and 0.42 at $230 \mathrm{GHz}$ (Ladd et al. 2005). The data analysis was conducted with CLASS90 software. Emission was detected in the $\mathrm{HCO}^{+}(1-0), \mathrm{HNC}(1-0)$, and $\mathrm{N}_{2} \mathrm{H}^{+}(1-0)$ lines, which were used to detect high density regions within SDC 341.232-0.268. Their main parameters are included in Table 1.

\subsection{Images in the Infrared}

We used near- and mid-infrared (NIR, MIR) images from the Spitzer-IRAC archive at 4.5 and $8.0 \mu \mathrm{m}$ of the Galactic Legacy Infrared Mid-Plane Survey 
Extraordinaire (GLIMPSE) ${ }^{8}$ (Benjamin et al. 2003), and the Multiband Imaging Photometer for Spitzer (MIPS) image at $24 \mu \mathrm{m}$ from the MIPS Inner Galactic Plane Survey (MIPSGAL) ${ }^{9}$ (Carey et al. 2005) to delineate the IRDC and investigate their correlation with the EGO candidates.

To trace the cold dust emission we utilized farinfrared (FIR) images from the Herschel Space Observatory belonging to the Infrared GALactic (HiGAL) plane survey key program (Molinari et al. 2010). The data were obtained out in parallel mode with the instruments PACS (Poglitsch et al. 2010) at 70 and $160 \mu \mathrm{m}$, and SPIRE (Griffin et al. 2010) at 250,350 , and $500 \mu \mathrm{m}$. The angular resolutions for the five photometric bands spans from $8^{\prime \prime}$ to $35^{\prime \prime} \cdot 2$ for $70 \mu \mathrm{m}$ to $500 \mu \mathrm{m}$. Herschel Interactive Processing Environment (HIPE v12 ${ }^{10}$, Ott 2010) was used to reduce the data, with reduction scripts from standard processing. The data reduction and calibration (including zero-level and color correction) is described in detail in $\S 2.2$ of Cappa et al. (2016).

We also used images at $870 \mu \mathrm{m}$ from the APEX Telescope Large Area Survey of the Galaxy (ATLASGAL) (Schuller et al. 2009) with a beam size of $19^{\prime \prime} 2$. This survey covers the inner Galactic plane, with an $r m s$ noise in the range $0.05-0.07 \mathrm{Jy}$ beam $^{-1}$. The calibration uncertainty in the final maps is about $15 \%$.

\section{MOLECULAR CHARACTERIZATION OF THE IRDC}

\subsection{CO data: Morphological and Kinematical Description}

To visualize the spatial distribution of the molecular emission the upper panel of Figure 2 exhibits the ${ }^{13} \mathrm{CO}(2-1)$ brightness temperature distribution from $\approx-47.0$ to $-42.5 \mathrm{~km} \mathrm{~s}^{-1}$ in steps of $0.3 \mathrm{~km} \mathrm{~s}^{-1}$. The most intense ${ }^{13} \mathrm{CO}(2-1)$ emission appears in the range -45.2 to $-44 \mathrm{~km} \mathrm{~s}^{-1}$. The bottom central panel of Figure 2 shows the average ${ }^{13} \mathrm{CO}(2-1)$ emission in the range -47.0 to $-42.8 \mathrm{~km} \mathrm{~s}^{-1}$, revealing two molecular clumps centered at RA, Dec. $(\mathrm{J} 2000)=(16: 52: 37.06,-44: 28: 13.9)($ Clump A $)$ and RA, Dec. $(\mathrm{J} 2000)=(16: 52: 29.72,-44: 28: 40.1)$ (Clump B). The coincidence of the clumps with

\footnotetext{
${ }^{8}$ http://irsa.ipac.caltech.edu/data/SPITZER/ GLIMPSE/

${ }^{9}$ http://irsa.ipac.caltech.edu/data/SPITZER/ MIPSGAL/

${ }^{10}$ HIPE is a joint development by the Herschel Science Ground Segment Consortium, consisting of ESA, the NASA Herschel Science Center, and the HIFI, PACS and SPIRE consortia members, see http://herschel.esac.esa. int/HerschelPeople.shtml.
}

EGO 1, 2, and 4 (indicated with crosses in this figure) is clear. The effective radii of the clumps are $377^{\prime \prime} 6$ and $455^{\prime \prime} 6$ for Clump $A$ and Clump B, respectively, as obtained taking into account the average ${ }^{13} \mathrm{CO}(2-1)$ emission higher that $6.2 \mathrm{~K}$. Finally, the bottom left and right panels of the same figure display the $\mathrm{C}^{18} \mathrm{O}(2-1)$ and ${ }^{12} \mathrm{CO}(2-1)$ average emission in velocity intervals similar to that in the image of ${ }^{13} \mathrm{CO}(2-1)$. Both clumps $\mathrm{A}$ and $\mathrm{B}$ are detected at $\mathrm{C}^{18} \mathrm{O}(2-1)$ revealing the existence of dense molecular gas in the clumps. The ${ }^{12} \mathrm{CO}(2-1)$ emission delineates the IRDC but the clumps are not well defined.

Figure 3 shows the averaged ${ }^{12} \mathrm{CO}(2-1)$, ${ }^{13} \mathrm{CO}(2-1)$, and $\mathrm{C}^{18} \mathrm{O}(2-1)$ spectra within the area corresponding to Clump $A$ and ClumpB. The rms noise of the averaged spectra is $0.019 \mathrm{~K}$ for Clump A, and 0.017 for Clump B. For Clump A, the ${ }^{12} \mathrm{CO}(2-1)$ line exhibits a multi-peak structure with components in the interval from -70 to $-20 \mathrm{~km} \mathrm{~s}^{-1}$, with the most intense features between -48 and $-35 \mathrm{~km} \mathrm{~s}^{-1}$. Three velocity componentes are detected within the latter velocity interval, peaking at $-46.6 \mathrm{~km} \mathrm{~s}^{-1},-42.2 \mathrm{~km} \mathrm{~s}^{-1}$, and $-36.8 \mathrm{~km} \mathrm{~s}^{-1}$. The ${ }^{13} \mathrm{CO}(2-1)$ and $\mathrm{C}^{18} \mathrm{O}(2-1)$ spectra peak at around $-44.0 \mathrm{~km} \mathrm{~s}^{-1}$, coincident with a minimum in the ${ }^{12} \mathrm{CO}(2-1)$ spectrum. Components outside this range will be analyzed in $\S 6.2$. For Clump B, the ${ }^{12} \mathrm{CO}(2-1)$ line also shows a multi-peak structure between -55 and $-25 \mathrm{~km} \mathrm{~s}^{-1}$, with the maximum at $-45.4 \mathrm{~km} \mathrm{~s}^{-1}$. The ${ }^{13} \mathrm{CO}(2-1)$ and $\mathrm{C}^{18} \mathrm{O}(2-1)$ profiles peak at $-44.0 \mathrm{~km} \mathrm{~s}^{-1}$.

Taking into account that the $\mathrm{C}^{18} \mathrm{O}(2-1)$ emission is generally optically thin, we adopted systemic velocities $\left(v_{\mathrm{sys}}\right)$ of $-44.0 \mathrm{~km} \mathrm{~s}^{-1}$ for Clump $A$ and Clump B. The adopted systemic velocity coincides with the velocity for EGO 2 and 4 reported by Yang et al. (2017) ( -44.6 and -44.9 , respectively) from methanol maser emision, and from masers by Chen et al. (2011). It is also compatible with the velocity of $\mathrm{NH}_{3}$ clouds identified by Purcell et al. (2012) at $-43.7 \mathrm{~km} \mathrm{~s}^{-1}$ at a position distant $15^{\prime \prime}$ from EGO 3, and from the CS(2-1) line emission obtained by Bronfman et al. (1996), who observed toward $[$ RA, Dec. $(J 2000)=(16: 52: 34.2,-44: 28: 36.0)]$, revealing the presence of high density regions $\left(n_{\text {crit }}=\right.$ $\left.3.0 \times 10^{5} \mathrm{~cm}^{-3}\right)$. Circular galactic rotation models predict that gas with velocities of $-44 \mathrm{~km} \mathrm{~s}^{-1}$ lies at the near kinematical distance of $3.6 \mathrm{kpc}$ (see, for example, Brand \& Blitz 1993). Adopting a velocity dispersion of $6 \mathrm{~km} \mathrm{~s}^{-1}$, the uncertainty in distance is $0.5 \mathrm{kpc}(15 \%)$. The distance coincides also with that of S 24 (Cappa et al. 2016) indicating that the IRDC belongs to the same complex. 


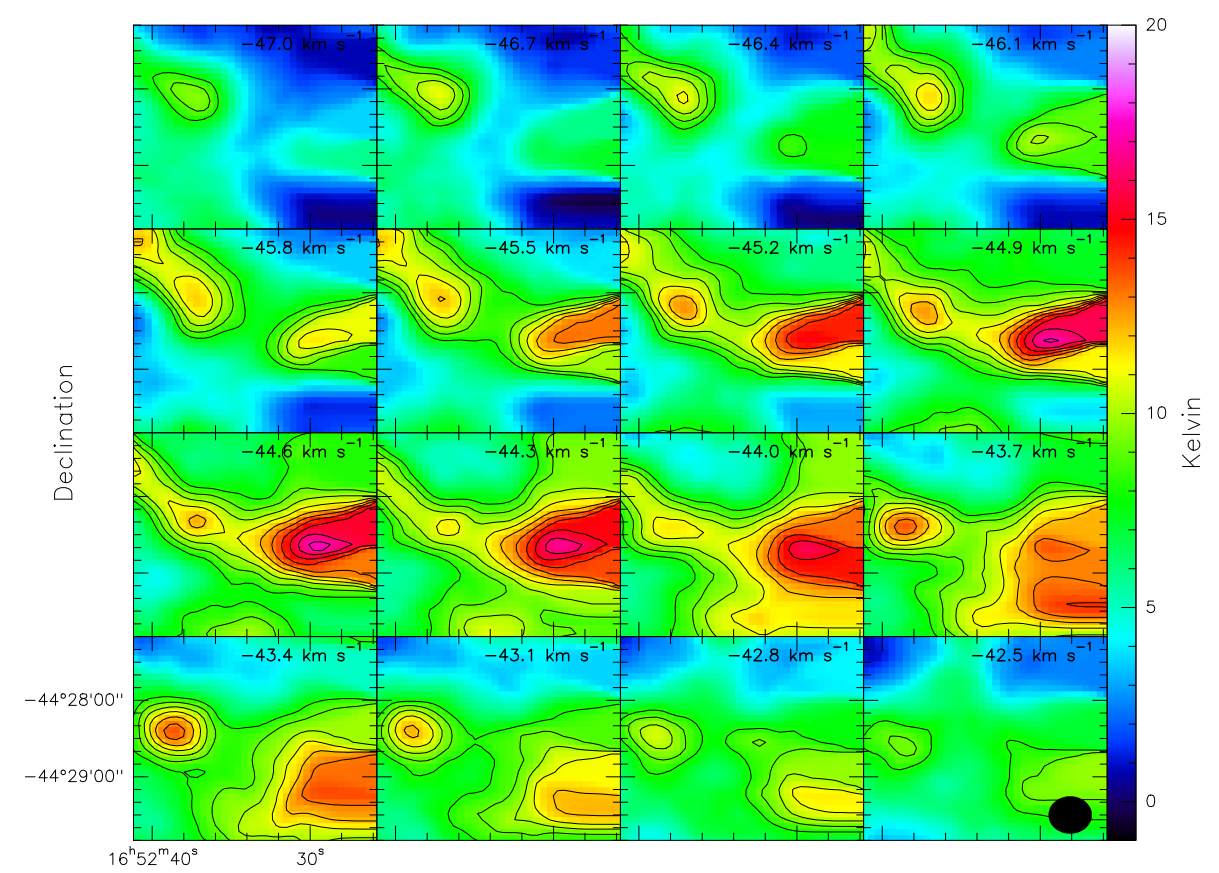

Right Ascension
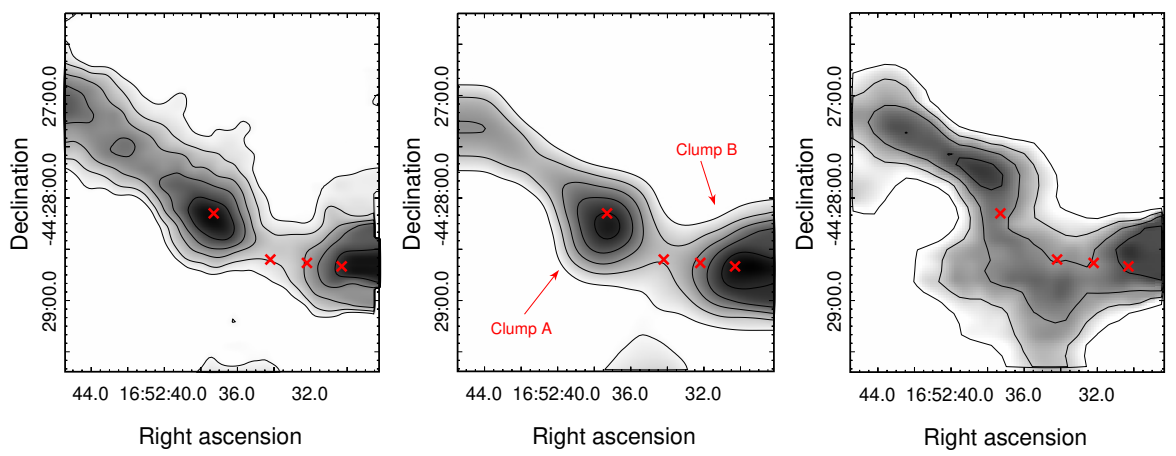

Fig. 2. Upper panel: $T_{\mathrm{mb}}$-maps of the ${ }^{13} \mathrm{CO}(2-1)$ emission within the velocity interval from -47.0 to $-42.5 \mathrm{~km} \mathrm{~s}^{-1}$ in steps of $0.3 \mathrm{~km} \mathrm{~s}^{-1}$. Contours range from 8 to $17 \mathrm{~K}$ in steps of $1 \mathrm{~K}$ in $T_{\mathrm{mb}}$. Lower left panel: Average $\mathrm{C}^{18} \mathrm{O}(2-1)$ emission in the range -47.2 to $-43.8 \mathrm{~km} \mathrm{~s}^{-1}$. Contours are $0.8,1.0,1.3,1.6$, and $2.0 \mathrm{~K}$. The red crosses mark the position of the EGOs. Lower central panel: Average ${ }^{13} \mathrm{CO}(2-1)$ emission in the range -47.0 to $-42.8 \mathrm{~km} \mathrm{~s}^{-1}$. Contours range from 6.2 to $9.4 \mathrm{~K}$ in steps of $0.8 \mathrm{~K}$. Lower right panel: Average ${ }^{12} \mathrm{CO}(2-1)$ emission in the range -48.2 to $-42.5 \mathrm{~km} \mathrm{~s}^{-1}$. Contours range from 10 to $13 \mathrm{~K}$ in steps of $1 \mathrm{~K}$. The color figure can be viewed online.

TABLE 2

PARAMETERS OF THE GAUSSIAN FITS FOR Clump A AND Clump B

\begin{tabular}{cccccc}
\hline & Line & $v$ & $\Delta \mathrm{v}$ & $T_{\text {peak }}$ & Area \\
& & $\mathrm{km} \mathrm{s}^{-1}$ & $\mathrm{~km} \mathrm{~s}^{-1}$ & $\mathrm{~K}$ & $\mathrm{~K} \mathrm{~km} \mathrm{~s}^{-1}$ \\
\hline \multirow{2}{*}{ Clump A } & ${ }^{13} \mathrm{CO}(2-1)$ & $-44.56(0.03)$ & $5.56(0.07)$ & 6.82 & $40.33(0.41)$ \\
& $\mathrm{C}^{18} \mathrm{O}(2-1)$ & $-44.23(0.02)$ & $4.46(0.05)$ & 1.37 & $6.49(0.06)$ \\
& ${ }^{13} \mathrm{CO}(2-1)$ & $-43.85(0.01)$ & $2.56(0.08)$ & 9.52 & $25.96(0.18)$ \\
& $\mathrm{C}^{18} \mathrm{O}(2-1)$ & $-43.70(0.01)$ & $2.61(0.03)$ & 2.46 & $6.84(0.07)$ \\
\hline
\end{tabular}




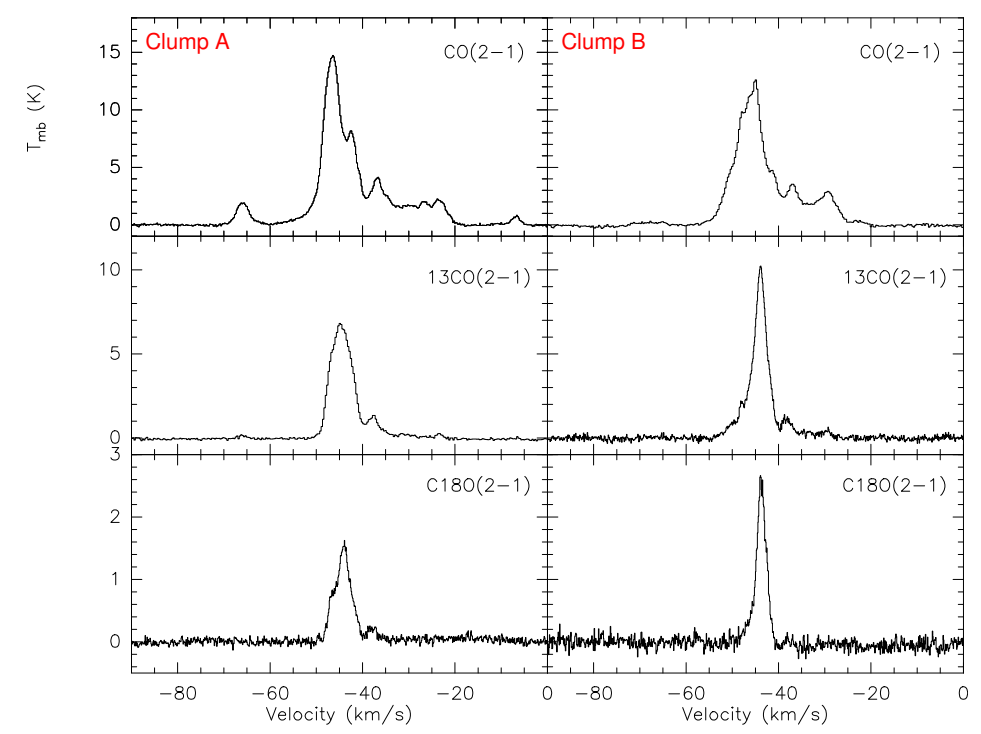

Fig. 3. Averaged ${ }^{12} \mathrm{CO}(2-1),{ }^{13} \mathrm{CO}(2-1)$ and $\mathrm{C}^{18} \mathrm{O}(2-1)$ spectra towards Clump $A$ (left) and Clump B(right).

TABLE 3

DERIVED PARAMETERS OF THE MOLECULAR COMPONENTS

\begin{tabular}{ccccccccccc}
\hline & $\tau_{13}$ & $\tau_{18}$ & $\begin{array}{c}T_{\text {exc }} \\
\mathrm{K}\end{array}$ & $\begin{array}{c}N\left({ }^{13} \mathrm{CO}\right) \\
10^{16} \mathrm{~cm}^{-2}\end{array}$ & $\begin{array}{c}N\left(\mathrm{H}_{2}\right) \\
10^{22} \mathrm{~cm}^{-2}\end{array}$ & $\begin{array}{c}r \\
\mathrm{pc}\end{array}$ & $\begin{array}{c}M\left(\mathrm{H}_{2}\right) \\
M_{\odot}\end{array}$ & $\begin{array}{c}n_{\mathrm{H} 2} \\
10^{4} \mathrm{~cm}^{-3}\end{array}$ & $\begin{array}{c}M_{\text {vir }} \\
M_{\odot}\end{array}$ & $\frac{M_{\text {vir }}}{M\left(\mathrm{H}_{2}\right)}$ \\
\hline Clump A & 3.4 & 0.6 & 11.5 & 14.20 & 7.1 & 0.7 & $2400 \pm 960$ & $2.6 \pm 1.3$ & $1000-1670$ & $0.41-0.69$ \\
Clump B & 1.1 & 0.1 & 14.4 & 4.12 & 2.06 & 0.9 & $1200 \pm 480$ & $0.5 \pm 0.2$ & $750-1240$ & $0.62-1.0$ \\
\hline
\end{tabular}

\subsection{Parameters Derived From CO Data}

Bearing in mind that the spatial distribution of the ${ }^{13} \mathrm{CO}(2-1)$ and $\mathrm{C}^{18} \mathrm{O}(2-1)$ emission coincides with the IRDC, we used these lines to estimate the main parameters of the molecular gas assuming local thermodynamic equilibrium (LTE). All the calculations were carried out from the Gaussian fits to the lines averaged in the area of Clump A and Clump B shown in Table 2. The columns list the central velocity of each component $v$, the full-width at halfmaximum, the peak temperature, and the integrated emission. We applied the following expression to estimate optical depths (e.g. Scoville et al. 1986):

$$
\frac{T_{\mathrm{mb}}^{13}}{T_{\mathrm{mb}}^{18}}=\frac{1-e^{-\tau^{13}}}{1-e^{-\tau^{13} / 7.6}},
$$

where $\tau^{13}$ is the optical depth of the ${ }^{13} \mathrm{CO}(2-1)$ gas and $7.6=\left[{ }^{13} \mathrm{CO}(2-1)\right] /\left[\mathrm{C}^{18} \mathrm{O}(2-1)\right]$ (Sanhueza et al. $2010)$ is the isotope abundance ratio. The ${ }^{13} \mathrm{CO}(2-1)$ optical depths are indicated in Column 2 of Table 3. To estimate the $\mathrm{C}^{18} \mathrm{O}(2-1)$ optical depth we used

$$
\tau_{18}=\frac{1}{7.6} \tau_{13}\left(\frac{\Delta \mathrm{v}_{13}}{\Delta \mathrm{v}_{18}}\right)\left(\frac{\nu^{13}}{\nu^{18}}\right)^{2}
$$

where $\Delta \mathrm{v}$ is the full-width at half-maximum of the $\mathrm{C}^{18} \mathrm{O}(2-1)$ and ${ }^{13} \mathrm{CO}(2-1)$ profiles. The results are listed in Column 3 of Table 3. Bearing in mind that ${ }^{13} \mathrm{CO}(2-1)$ is moderately optically thick we calculated the excitation temperature from the ${ }^{13} \mathrm{CO}(2-1)$ line using

$$
T_{\mathrm{exc}}=\frac{T^{*}}{\ln \left[\left(\frac{T_{\mathrm{mb}}^{13}}{T^{*}}+\frac{1}{\left.\exp \left(T^{*} / T_{\mathrm{bg}}\right)-1\right)}\right)^{-1}+1\right]},
$$

where $T^{*}=\frac{h \nu}{k}, \nu$ is the frequency of the ${ }^{13} \mathrm{CO}(2-1)$ line and $T_{\mathrm{bg}}=2.7 \mathrm{~K}$. Values estimated for $T_{\text {exc }}$ are listed in Table 3.

The column density for ${ }^{13} \mathrm{CO}(2-1)$ was derived using Rohlfs \& Wilson (2004)

$$
\begin{aligned}
N\left({ }^{13} \mathrm{CO}\right)= & 2.4 \times 10^{14} \exp \left(\frac{T^{*}}{T_{\mathrm{exc}}}\right) \\
& \times \frac{T_{\text {exc }}}{1-e^{-T^{*} / T_{\mathrm{exc}}}} \int \tau_{13} d \mathrm{v} .
\end{aligned}
$$

In this case we used the approximation for $\tau_{13}>1$,

$$
T_{\text {exc }} \int \tau_{13} d \mathrm{v} \simeq \times \frac{\tau^{13}}{1-\exp \left(-\tau^{13}\right)} \int T_{\mathrm{mb}}^{13} d \mathrm{v} .
$$




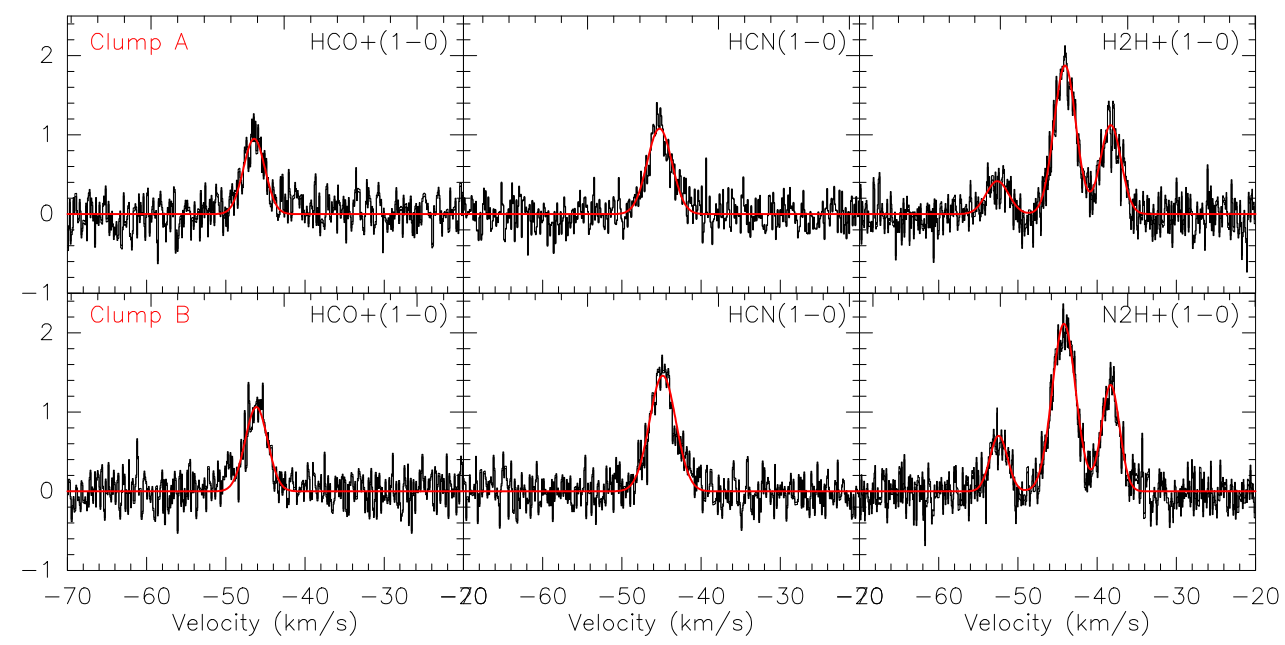

Fig. 4. $\mathrm{HCO}^{+}(1-0), \mathrm{HNC}(1-0)$ and $\mathrm{N}_{2} \mathrm{H}^{+}(1-0)$ profiles towards Clump $A$ (top) and Clump B (bottom). The color figure can be viewed online.

This approximation helps to eliminate to some extent optical depth effects. The integral was evaluated as $T_{\text {mean }-m b} \times \Delta \mathrm{v}$, where $T_{\text {mean-mb }}$ is equal to the average $T_{m b}$ within the area of each clump.

To estimate $N\left(\mathrm{H}_{2}\right)$ (Column 6 in Table 3) we adopted an abundance $\left[\mathrm{H}_{2}\right]\left[{ }^{13} \mathrm{CO}\right]=5 \times 10^{5}$ (Dickman 1978). Then, the molecular mass (Column 9 in Table 3) was calculated from the equation

$$
M\left(H_{2}\right)=\left(m_{\text {sun }}\right)^{-1} \mu m_{\mathrm{H}} A N\left(H_{2}\right) d^{2},
$$

where $m_{\text {sun }}=2 \times 10^{33} \mathrm{~g}$ is the solar mass, the mean molecular weight $\mu=2.76$ (which includes a relative helium abundance of $25 \%$ by mass, Allen 1973), and $m_{\mathrm{H}}$ is the hydrogen atomic mass. In this expression $d$ is the distance, $N\left(H_{2}\right)$ is the $\mathrm{H}_{2}$ column density, and $A$ is the area of the source in $\mathrm{cm}^{-2}$. The effective radius of each clump, as seen in the ${ }^{13} \mathrm{CO}(2-1)$ line, and the volume densities are listed in Colums 7 and 9 in Table 3.

The ambient volume densities, $n_{\mathrm{H}_{2}}$, was calculated assuming a spherical geometry for the clumps, using the formula

$$
n_{\mathrm{H}_{2}}=\frac{M\left(H_{2}\right)}{\frac{4}{3} \pi r^{3} \mu m_{H}} .
$$

Ambient densities are listed in Column 9 of Table 3. The parameters calculated in this work agree with Bergin \& Tafalla (2007).

The virial mass can be determined following MacLaren et al. (1988):

$$
\frac{M_{\mathrm{vir}}}{M_{\odot}}=k_{2}\left[\frac{r}{\mathrm{pc}}\right]\left[\frac{\Delta \mathrm{v}^{2}}{\mathrm{kms}^{-1}}\right]
$$

where $r$ and $\Delta \mathrm{v}$ are the radius of the region and the velocity width measured from the Gaussian fit of the $\mathrm{C}^{18} \mathrm{O}(2-1)$ emission, and $k_{2}$ depends on the geometry of the ambient gas in the region, being 190 or 126 according to $\rho \propto r^{-1}$ or $\rho \propto r^{-2}$, respectively. $M_{\mathrm{vir}}$ values are listed in Column 10 in Table 3. The ratios $M_{\text {vir }} / M\left(H_{2}\right)$ suggest that both clumps may collapse to form new stars; since the virial mass value is smaller than the LTE mass value this clump does not have enough kinetic energy to stop the gravitational collapse.

Uncertainties in both the molecular mass derived using LTE conditions, $M\left(\mathrm{H}_{2}\right)$, and the virial mass $M_{\text {vir }}$ are affected by the distance indetermination (15\%) yielding a $30 \%$ error in $M\left(\mathrm{H}_{2}\right)$ and $15 \%$ in $M_{\mathrm{vir}}$. Inaccuracies in the borders of the clumps originate errors in their sizes and thus additional uncertainties in the masses, suggesting errors of $40 \%$ in $M\left(\mathrm{H}_{2}\right)$. Virial masses are not free of uncertainties, because of the existence of magnetic field support that may lead to an overestimate of the derived values (see MacLaren et al. 1988), and because of the unknown density profile of the clump.

\subsubsection{Analysis of the MALT90 Data}

The MALT90 data cubes of this region show emission of $\mathrm{HCO}^{+}(1-0), \mathrm{HNC}(1-0)$, and $\mathrm{N}_{2} \mathrm{H}^{+}(1-0)$ molecules, which are the most often detected molecules towards IRDCs (Sanhueza et al. 2012; Rathborne et al. 2016). These molecules have critical densities of $2 \times 10^{5}, 3 \times 10^{5}$ and $3 \times 10^{5} \mathrm{~cm}^{-3}$, respectively (Sanhueza et al. 2012), allowing us to infer a lower limit for the density of the clumps. These val- 


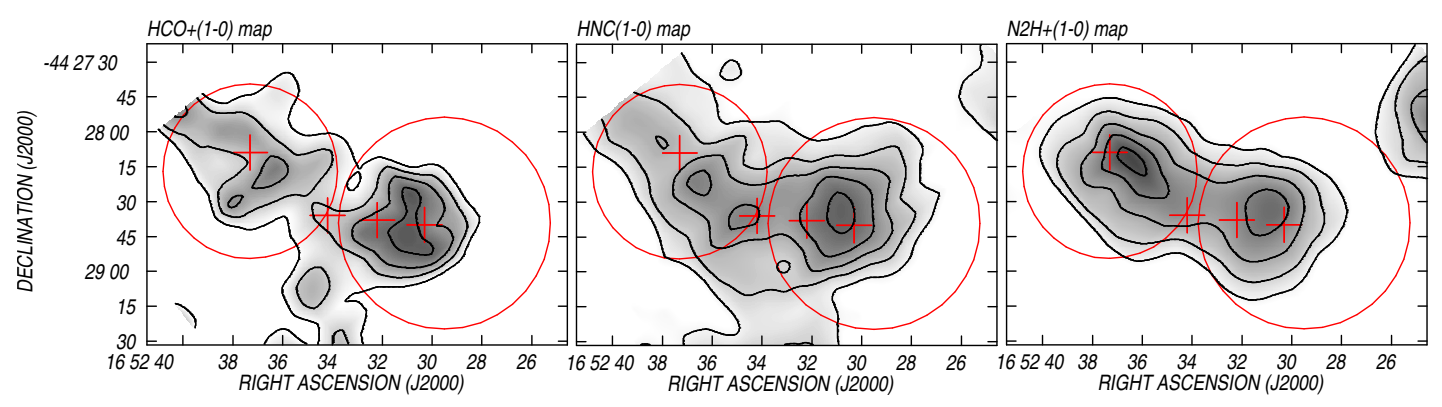

Fig. 5. $\mathrm{HCO}^{+}(1-0)$ (levs: $0.65,0.7,0.8$ and $\left.0.9 \mathrm{~K}\right), \mathrm{HNC}(1-0)($ levs: $0.7,0.85,1.0$ and $1.15 \mathrm{~K})$ and $\mathrm{N}_{2} \mathrm{H}^{+}(1-0)($ levs: $0.8,1.0,1.2$ and $1.4 \mathrm{~K})$ maps. Red circles have radii equal to the effective radii of the clumps as seen in ${ }^{13} \mathrm{CO}(2-1)$, and are located at their position. Crosses mark the location of the EGO candidates. The color figure can be viewed online.

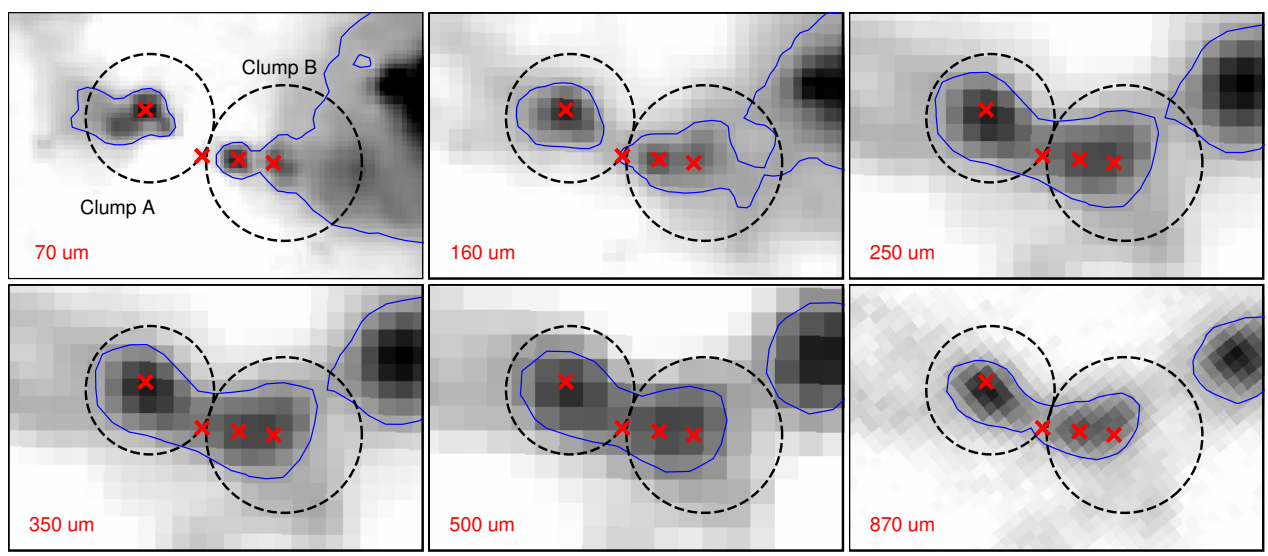

Fig. 6. FIR view of SDC 341.232-0.268 from Herschel $(70-500 \mu \mathrm{m})$ and ATLASGAL data. The blue circles have the same meaning as in Figure 5 and show the areas where the flux densities were integrated. The crosses mark the position of the EGOs. Contours correspond to $20 \mathrm{rms}$. The color figure can be viewed online.

ues are higher than the $\mathrm{H}_{2}$ ambient densities derived from CO lines (see Table 3 ).

Spectra of these lines, averaged over the area of the clumps, are shown in Figure 5. By applying a Gaussian fitting to the $\mathrm{HCO}^{+}$and $\mathrm{HNC}$ lines, a mean velocity of $-46 \mathrm{~km} \mathrm{~s}^{-1}$ was derived. For the case of the $\mathrm{N}_{2} \mathrm{H}^{+}$line, a multi Gaussian function was applied to take into account hyperfine structure. This molecule has seven hyperfine components in two blended groups of three lines (groups 2 and 3 ) and one isolated component (group 1). The rest frequencies of groups 1, 2 and 3 are 93.176, 93.173, and $93.171 \mathrm{GHz}$, respectively. The mean velocity is $-44 \mathrm{~km} \mathrm{~s}^{-1}$. The velocities of these lines coincide with the systemic velocities derived from $\mathrm{CO}$ data.

These tracers of dense gas provide slightly different information: $\mathrm{HCO}^{+}$often shows infall signatures and outflow wings (e.g. Rawlings et al. 2004; Fuller et al. 2005). However, in our case no signs of infall can be identified. HNC is specially preponderant in cold gas and is a commonly used tracer of dense gas in molecular clouds. Finally, $\mathrm{N}_{2} \mathrm{H}^{+}$is more resistant to freeze-out on grains than carbon-bearing species like CO.

Figure 5 shows the $\mathrm{HCO}^{+}(1-0), \mathrm{HNC}(1-0)$ and $\mathrm{N}_{2} \mathrm{H}^{+}(1-0)$ maps, integrated from -47.6 to $-44.0 \mathrm{~km} \mathrm{~s}^{-1},-46.7$ to $-43.1 \mathrm{~km} \mathrm{~s}^{-1}$, and -47 to $-41.4 \mathrm{~km} \mathrm{~s}^{-1}$, respectively. Both clumps are detected in the three molecular lines indicating mean densities of $10^{5} \mathrm{~cm}^{-3}$. Carbon-bearing species, like $\mathrm{CO}$, tend to disappear from the gas phase in the high density centers of the cores, while nitrogen-bearing species like $\mathrm{N}_{2} \mathrm{H}^{+}$survive almost unaffected up to much higher densities. This is evidenced in the fact that the spacial distribution of the $\mathrm{N}_{2} \mathrm{H}^{+}$emission is similar to that of dust continuum emission (see Figure 6).

Following Purcell et al. (2009) we estimate the $\mathrm{N}_{2} \mathrm{H}^{+}$optical depth and column density. Assuming the line widths of the individual hyperfine components are all equal, the integrated intensities of the three blended groups should be in the ratio of 1:5:3 
under optically thin conditions. The optical depth can then be derived from the ratio of the integrated intensities of any group using the following equation:

$$
\frac{\int T_{\mathrm{mb}, 1} d \mathrm{v}}{\int T_{\mathrm{mb}, 2} d \mathrm{v}}=\frac{1-\exp \left(-\tau_{1}\right)}{1-\exp \left(-\tau_{2}\right)}=\frac{1-\exp \left(-\tau_{1}\right)}{1-\exp \left(-a \tau_{1}\right)},
$$

where $a$ is the expected ratio of $\tau_{1} / \tau_{2}$ under optically thin conditions.

To determine the optical depth we used only the intensity ratio of group 1 /group 2, as Caselli et al. (1995) report anomalous excitation of the $\mathrm{F}_{1}, \mathrm{~F}=$ $1,0 \rightarrow 1,1$ and $1,2 \rightarrow 1,2$ components (in group 3 ). Thus we obtained $\tau_{1}=0.11$ and 0.19 for Clump $A$ and Clump B, respectively.

Based on the expression for $T_{\mathrm{mb}}$ given by Rohlfs \& Wilson (2004), the excitation temperature for $\mathrm{N}_{2} \mathrm{H}^{+}$can be calculated with the following formula:

$T_{\text {exc }}=4.47 / \ln \left[1+\left(\frac{T_{\mathrm{mb}}}{4.47(1-\exp (-\tau))}+0.236\right)^{-1}\right]$,

and the column densities can be derived using (Chen et al. 2013):

$$
\begin{aligned}
N\left(\mathrm{~N}_{2} \mathrm{H}^{+}\right)= & \frac{3 k W}{8 \pi^{3} \nu S \mu^{2}}\left(\frac{T_{\mathrm{exc}}}{T_{\mathrm{exc}}-T_{\mathrm{bg}}}\right)\left(\frac{\tau}{1-\exp (-\tau)}\right) \\
& \times Q\left(T_{\mathrm{exc}}\right) \exp \left(E_{u} / k T_{\mathrm{exc}}\right)
\end{aligned}
$$

where $k$ is the Boltzmann constant, $W$ is the observed line integrated intensity (obtained from a Gaussian fit), $\nu$ is the frequency of the transition, and $S \mu^{2}$ is the product of the total torsion-rotational line strength and the square of the electric dipole moment. $T_{\text {exc }}$ and $T_{\mathrm{bg}}$ are the excitation temperature and background brightness temperature, respectively. $E_{u} / k$ is the upper level energy in $\mathrm{K}$, $Q\left(T_{\text {exc }}\right)$ is the partition function at temperature $T_{\text {exc }}$ and $\tau$ is the optical depth. For group 1 the values of $\nu, S \mu^{2}$ and $E_{u} / k$ are $93176.2526 \mathrm{MHz}, 12.42 \mathrm{D}^{2}$ and $4.47 \mathrm{~K}$, respectively. These values were taken from the SPLATALOGUE catalogue ${ }^{11}$.

We derive $T_{\mathrm{exc}}=17.4$ and $12.5 \mathrm{~K}$, and $N\left(\mathrm{~N}_{2} \mathrm{H}^{+}\right)=8.7 \times 10^{13}$ and $8.1 \times 10^{13}$ for Clump $A$ and Clump B, respectively. Considering an abundance $\left[\mathrm{N}_{2} \mathrm{H}^{+}\right] /\left[\mathrm{H}_{2}\right]=5 \times 10^{-10}$ for dark molecular clouds (Ohishi et al. 1992) we obtain $\mathrm{H}_{2}$ column densities of 1.7 and $1.6 \times 10^{23}$ for Clump $A$ and Clump B, respectively. These column densities are higher than those obtained from $\mathrm{CO}$ calculations. Ambient densities are $n_{H_{2}} \approx 1.7 \times 10^{5} \mathrm{~cm}^{-3}$

\footnotetext{
${ }^{11}$ SPLATALOGUE at http://www.splatalogue.net/
}

for each clump with an effective radii in $\mathrm{N}_{2} \mathrm{H}^{+}$of $0.53 \mathrm{pc}$. This difference may be explained by considering that the nitrogen-bearing species survive almost unaffected up to much larger densities that carbon-bearing molecules.

\section{WARM AND COLD DUST DISTRIBUTION}

Figure 6 shows Herschel and ATLASGAL maps (in original resolution) of the SDC 341.232-0.268 region. At $\lambda<160 \mu \mathrm{m}$ the beam resolution allows to identify the emission associated with EGOs 1, 2, and 4. Warm dust coincident with the EGOs is also revealed by the emission in $24 \mu \mathrm{m}$, which can be seen in Figure 1. The presence of this emission allows us to classify the clumps as "active", according to the classification of Chambers et al. (2009). These authors proposed an evolutionary sequence in which "quiescent" clumps (containing neither IR indicator) evolve into "intermediate" (containing either a "green fuzzy" or a $24 \mu \mathrm{m}$ point source, but not both), "active" (caracterized by the presence of a "green fuzzy" coincident with an embedded $24 \mu \mathrm{m}$ source, such as those observed toward EGOs 1, 2, and 4), and "red" clumps (dominated by $8 \mu \mathrm{m}$ emission, which contains PAH features). At $\lambda>160 \mu \mathrm{m}$ two dust clumps are detected superimposed onto more extended submillimeter emission, indicating dust related to Clump A and Clump B.

The characterization of the dust properties of these clumps is limited by the resolution of FIR data. We study the integrated dust properties of each clump from the spectral energy distribution (SED) using the Herschel (160 up to $500 \mu \mathrm{m}$ ) and ATLASGAL maps convolved at a common beam resolution of the $500 \mu \mathrm{m}$ map $\left(36^{\prime \prime}\right)$. The flux densities are obtained from a circular aperture photometry integration (radius of $37^{\prime \prime} 6$ and $45{ }^{\prime \prime} 6$ for Clump $A$ and Clump B, respectively), and subtracting a background level computed from a rectangular region (width $=52$ '.5 and height $25^{\prime \prime} .8$ in the north of the clumps). For flux uncertainty estimation, we consider the standard deviation of surface brightness in the background region and the flux calibration uncertainties. For each clumps, the final SED is depicted in Figure 7 and the fluxes and their error bars are listed in Table 4. We perform a thermal dust fit to the data considering a single-component modified blackbody (grey-boby), which depends on the optical depth, the emissivity spectral index $\left(\beta_{d}\right)$ and the dust temperature $\left(T_{d}\right)$. In the optically thin regime, the best fitting provides similar dust temperature and emissivity for both clumps, with values of 

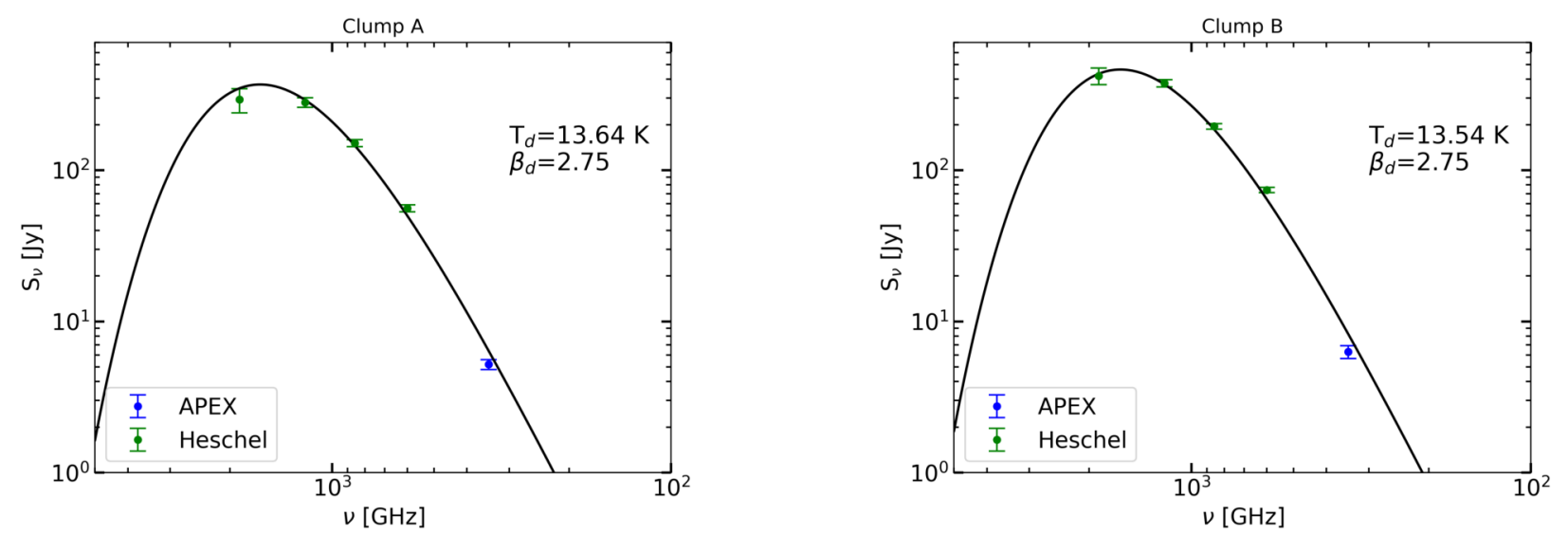

Fig. 7. Spectral energy distributions (SEDs) for Clump A (left) and Clump B (right), obtained from the fluxes at 160 , 250, 350, and $500 \mu \mathrm{m}$ from Herschel, and $870 \mu \mathrm{m}$ from ATLASGAL. The color figure can be viewed online.

TABLE 4

MEASURED FLUXES AND DERIVED PARAMETERS OF THE FIR CLUMPS

\begin{tabular}{ccccccccc}
\hline & $\mathrm{S}_{160}$ & $\mathrm{~S}_{250}$ & $\mathrm{~S}_{350}$ & $\mathrm{~S}_{500}$ & $\mathrm{~S}_{870}$ & $T_{d}$ & $M_{\text {dust+gas }}$ & $\begin{array}{c}n_{\mathrm{H}_{2}} \\
{[\mathrm{Jy}]} \\
{[\mathrm{Jy}]}\end{array}$ \\
{$[\mathrm{Jy}]$} & {$[\mathrm{Jy}]$} & {$[\mathrm{Jy}]$} & {$[\mathrm{Jy}]$} & {$[\mathrm{K}]$} & {$\left[M_{\odot}\right]$} & {$\left[\mathrm{cm}^{-3}\right]$} \\
\hline Clump $A$ & $293 \pm 53$ & $281 \pm 21$ & $151 \pm 8$ & $56 \pm 3$ & $5.2 \pm 0.4$ & $13.6 \pm 0.47$ & $1536 \pm 444$ & $1.5 \pm 0.7$ \\
Clump B & $421 \pm 53$ & $376 \pm 21$ & $195 \pm 8$ & $74 \pm 3$ & $6.3 \pm 0.6$ & $13.5 \pm 0.47$ & $2088 \pm 600$ & $1.4 \pm 0.6$ \\
\hline
\end{tabular}

$T_{d}=13.5 \pm 0.47 \mathrm{~K}$ and $\beta_{d}=2.7$. These dust temperatures are of the order of those found by Guzmán et al. (2015) (18.6 \pm 0.2$)$ for proto-stellar clumps.

For Clump A and ClumpB, the total mass ( $\left.M_{\text {dust+gas }}\right)$ was calculated from the optical depth of the dust obtained from the fit, using the following expression

$$
M_{d u s t+g a s}=\frac{\tau_{\nu_{f i t}}}{\kappa_{870}}\left(\frac{345 \mathrm{GHz}}{\nu_{f i t}}\right)^{\beta_{d}} d^{2} R_{\mathrm{d}}
$$

where $\tau_{\nu_{\text {fit }}}$ is the dust optical depth ( $=10^{-26} \times$ fit amplitude), $\nu_{f i t}=1 \mathrm{~Hz}$ is the frequency of the fit, $R_{\mathrm{d}}=100$ is the typical gas-to-dust ratio, $d$ is the distance and $\kappa_{870}=1.00 \mathrm{~cm}^{-2} \mathrm{gr}^{-1}$ is the dust opacity at $345 \mathrm{GHz}$ (Ossenkopf \& Henning 1994). The mass uncertainties were computed propagating the error bars for dust optical depth and distance. Additional errors may result from taking different values for the gas-to-dust ratio (in our galaxy typical values are between 100 and 150).

The results are listed in Table 4, which includes the derived masses of the clumps and their volume densities. Uncertainties in masses and ambient densities are about $35 \%$ and $60 \%$, respectively. The total masses derived from molecular gas and dust emission are in good agrement (within errors).

\section{STAR FORMATION}

\subsection{Search for Additional YSOs Coincident with the IRDC}

To investigate the coincidence of the IRDC with other candidate young stellar objects (YSOs), we analyze the characteristics of the point sources in the WISE and Spitzer catalogues (Wright et al. 2010; Benjamin et al. 2003) projected in the region. In Figure 8 we mark the positions of Class I and II YSO candidates identified in the area of the molecular clumps. An inspection of the figure reveals that EGOs 1,3 , and 4 coincide with identified YSOs.

To study in some detail the nature of the EGOs we plotted their spectral energy distributions (SEDs) using the Robitaille's SED fitting tool (Robitaille et al. 2007). To perform the analysis we were able to use available data from 2MASS, IRAC-GLIMPSE, and MIPSGAL at $24 \mu \mathrm{m}$ only. The obtained SEDs are shown in Figure 9. Clearly, EGO 1, 3 and 4 have characteristics of YSOs, and as seen in Figure 8 coincide with additional evidence of star formation. As regards EGO 2, the fit is not good; it does not coincide with other sources or with the molecular clumps. So, its status is doubtful. In Table 5 we display the main parameters obtained from the SEDs. Column 1 gives the name of the source; Columns 2 
TABLE 5

PHYSICAL PARAMETERS OBTAINED FROM THE SEDS FOR THE EGOS

\begin{tabular}{ccccccc}
\hline & Age & $M_{\text {stellar }}$ & $M_{\text {env }}$ & $\dot{M}_{a c r}$ & $L$ & Stage \\
& $10^{4}$ & $\mathrm{M}_{\odot}$ & $\mathrm{M}_{\odot}$ & $10^{-4} M_{\odot} \mathrm{yr}^{-1}$ & $10^{2} L_{\odot}$ & \\
\hline EGO 1 & 0.13 & 2.1 & 0.7 & 0.1 & 1.3 & $0 / \mathrm{I}$ \\
EGO 2 & 545 & 3.9 & $8 \times 10^{-6}$ & 0 & 1.9 & $0 / \mathrm{I}$ \\
EGO 3 & 15.7 & 4.1 & 7.1 & 1.4 & 0.8 & $0 / \mathrm{I}$ \\
EGO 4 & 4.1 & 8.6 & 0.09 & 12.5 & 14.4 & $0 / \mathrm{I}$ \\
\hline
\end{tabular}

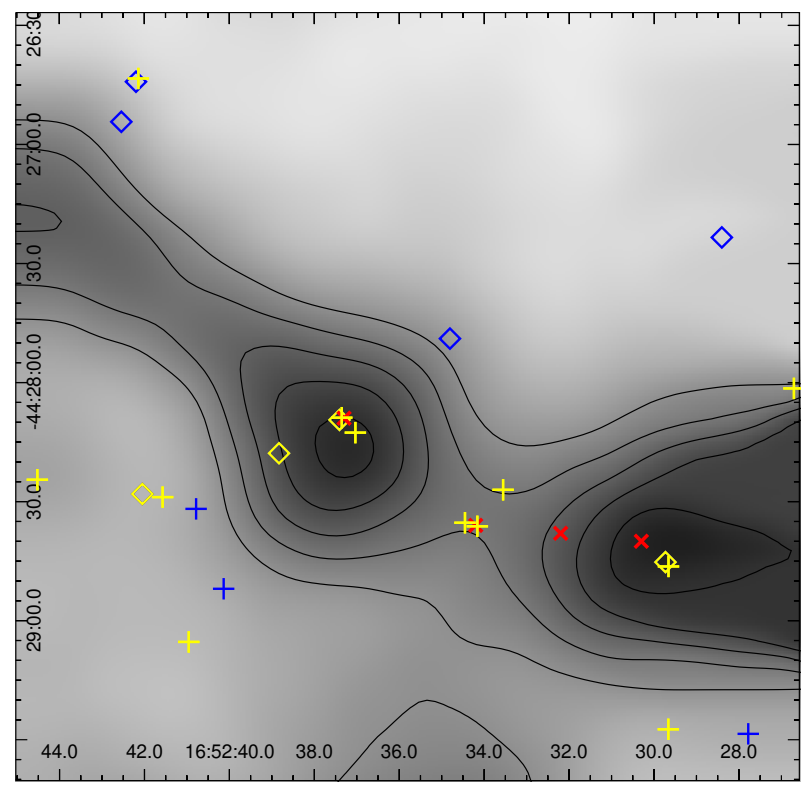

Fig. 8. Overlay of YSOs identified in the Spitzer and WISE catalogues and the molecular clumps in ${ }^{13} \mathrm{CO}(2-1)$. Contours have the same meaning as in Figure 2. Yellow and blue symbols show the position of Class I and Class II YSOs, while red crosses mark the location of EGOs. Plus signs correspond to Spitzer sources and diamonds to WISE ones. The color figure can be viewed online.

and 3 , the age and mass, $M_{\text {stellar, of the central }}$ source; Column 4, the mass of the envelope, $M_{\mathrm{env}}$; Column 5, the infall rate, $\dot{M}_{\text {acr }}$; and Column 6 , the total luminosity. To perform the SEDs we adopted $d=3.6 \pm 0.5 \mathrm{kpc}$ and a visual extinction of $3-4 \mathrm{mag}$.

Following Robitaille et al. (2007) an estimate of the evolutionary stage of the sources can be obtained based on the ratio of the infall rate and the mass of the central source. For the four candidate EGOs the ratio $\dot{M}_{\text {acr }} / M_{\text {stellar }}>10^{-6}$ indicates Stage $0 /$ I. The age of some of the sources suggests that they are still immersed in their envelopes. According to the fitting, EGO 4 would be the most massive object in the sample, and EGO 2 seems to be the most evolved one. These results should be taken with caution because of the limitations of the fitting tool that may arise from: (1) YSOs are complex 3d objects with slightly non-axisymmetric density structures, so the models are incorrect compared to actual density distributions; (2) there are often mixtures of sources and many objects appear as a single YSO, but they are often two or more objects; and (3) even in the case of an isolated YSO variability is an additional complication, since the data with which SEDs are usually constructed belong to different surveys that have been performed in different years and can produce discontinuities in the SEDs (Robitaille 2008; Deharveng et al. 2012; Offner et al. 2012).

As pointed out before, the emission at $24 \mu \mathrm{m}$, detected toward EGO 1, 3, and 4, suggests the existence of warm dust and embedded protostars (Jackson et al. 2008).

\subsection{Evidence for Inflow/Outflow Motions}

One way to visualize molecular outflows or infalls is to compare the optically thick ${ }^{12} \mathrm{CO}(2-1)$ emission line with the optically thin $\mathrm{C}^{18} \mathrm{O}(2-1)$ molecular line toward the candidate EGOs, as shown in Figure 10 for EGO 3 and EGO 4. The ${ }^{12} \mathrm{CO}(2-1)$ line presents a double peak structure with the blue shifted peak brighter than the red-shifted one, and a minimum at the systemic velocity, while the $\mathrm{C}^{18} \mathrm{O}(2-1)$ line presents a single peak centered on the absorption dip of the optically thick line. According to Chen et al. (2011) these spectra display characteristics typical of infall motions: a double-peak structure with the blue-shifted peak brighter than the red-shifted one, while an emission peak of an optically thin line appears centered on the absorption dip of the optically thick line. According to these authors, the infall motion is the only process that would consistently produce the blue profile asymmetry. The blue-shifted emission can be explained as due to high-excitation 

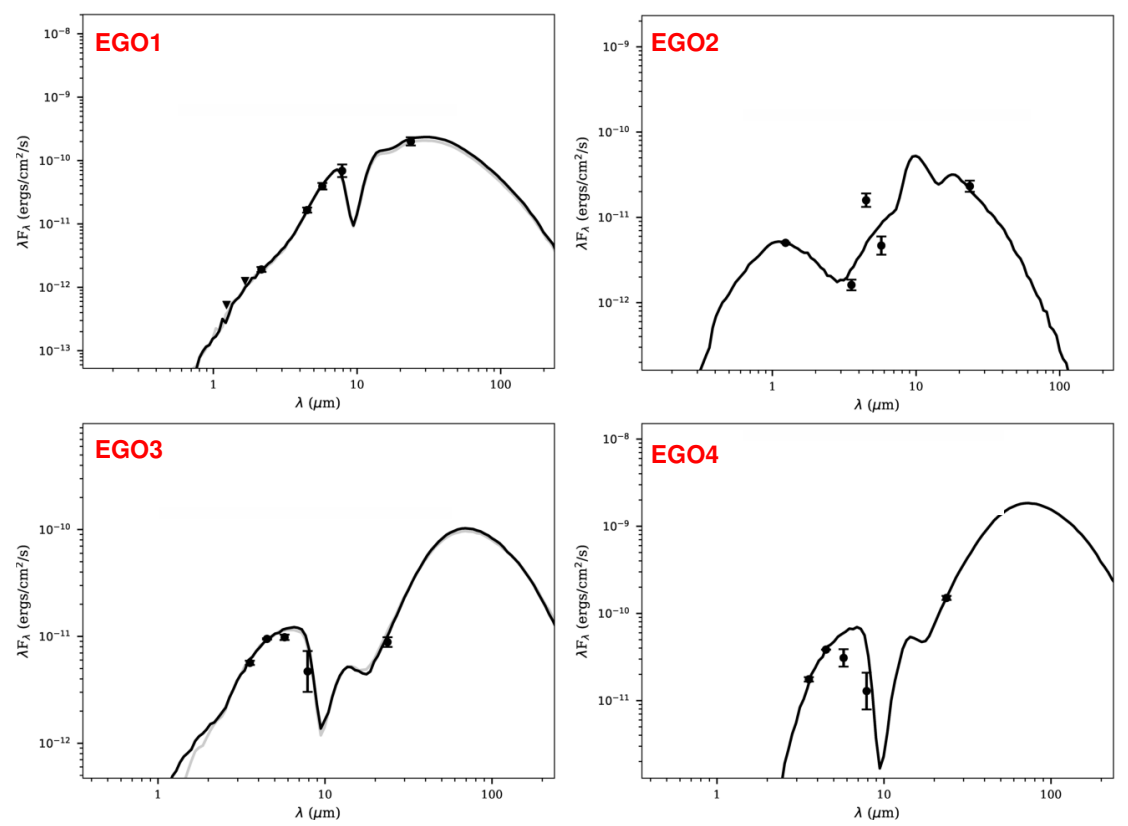

Fig. 9. Spectral energy distribution for the four candidate EGOs. The color figure can be viewed online.
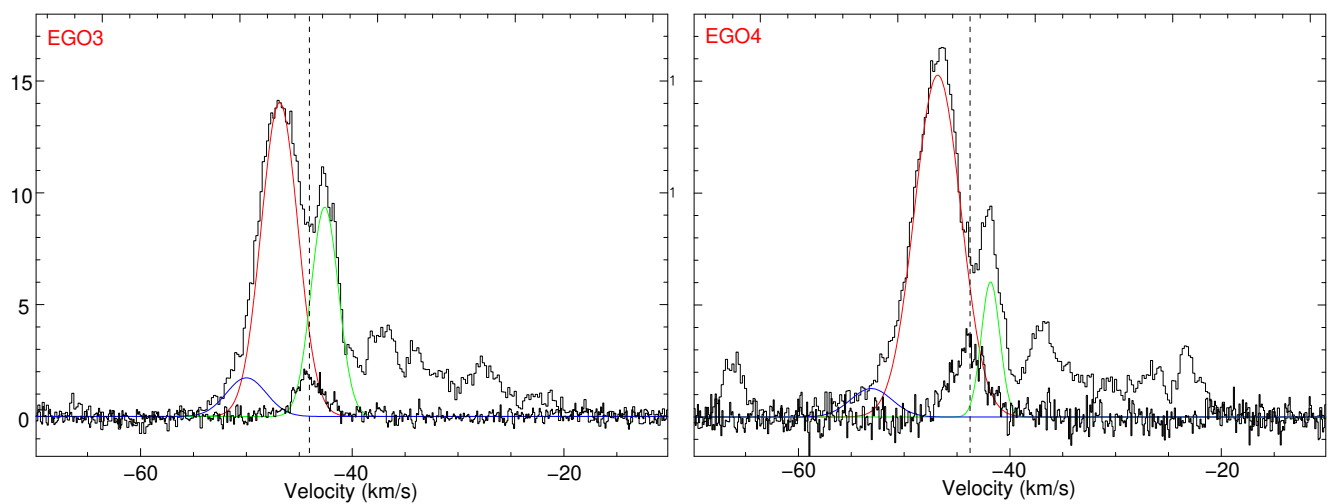

Fig. 10. ${ }^{12} \mathrm{CO}(2-1)$ and $\mathrm{C}^{18} \mathrm{O}(2-1)$ profiles in the direction to EGO 3 (left) and EGO 4 (right). In both pannels red and green lines correspond with Gaussian fits from the ${ }^{12} \mathrm{CO}(2-1)$ double peak, and blue lines indicates a Gaussian component fitting the possible blue outflow wings. The color figure can be viewed online.

approaching warm gas located on the far side of the center of contraction. The emission of this gas undergoes less extinction than the emission from the red-shifted receding nearside material, given that the excitation temperature of the molecules increases toward the center of the region (Zhou 1992). Thus, the spectra of EGO 3 and EGO 4 suggest the presence of infall motions.

Following Mardones et al. (1997), we use the asymmetry parameter $\delta \mathrm{v}$ (the velocity difference between the peaks of the optically thick line and the optically thin lines, normalized by the FWHM of the thin line), to quantify the blue asymmetry. The pa- rameter is defined as $\delta \mathrm{v}=\left(\mathrm{v}_{\text {thick }}-\mathrm{v}_{\text {thin }}\right) / \Delta \mathrm{v}_{\text {thin }}$. A statistically significant excess of blue asymmetric line profiles with $\delta \mathrm{v}<-0.25$ indicates that the molecular gas is falling into the clump. We consider the velocity and width of the $\mathrm{C}^{18} \mathrm{O}(2-1)$, from Table 2 , as $\mathrm{v}_{\text {thin }}$ and $\Delta \mathrm{v}_{\text {thin }}$, and calculate $\mathrm{v}_{\text {thick }}$ from the Gaussian fits of ${ }^{12} \mathrm{CO}(2-1)$ profiles $(-46.9$ and -45.3 from Clump $A$ and Clump B, respectively). We find, for both Clump $A$ and Clump $B \delta \mathrm{v} \approx-0.06$, which supports the infall hypothesis.

The interpretation presented above is supported by the $M_{\mathrm{vir}} / M\left(\mathrm{H}_{2}\right)$ ratio previously derived for the objects. As the classical virial equilibrium analysis 


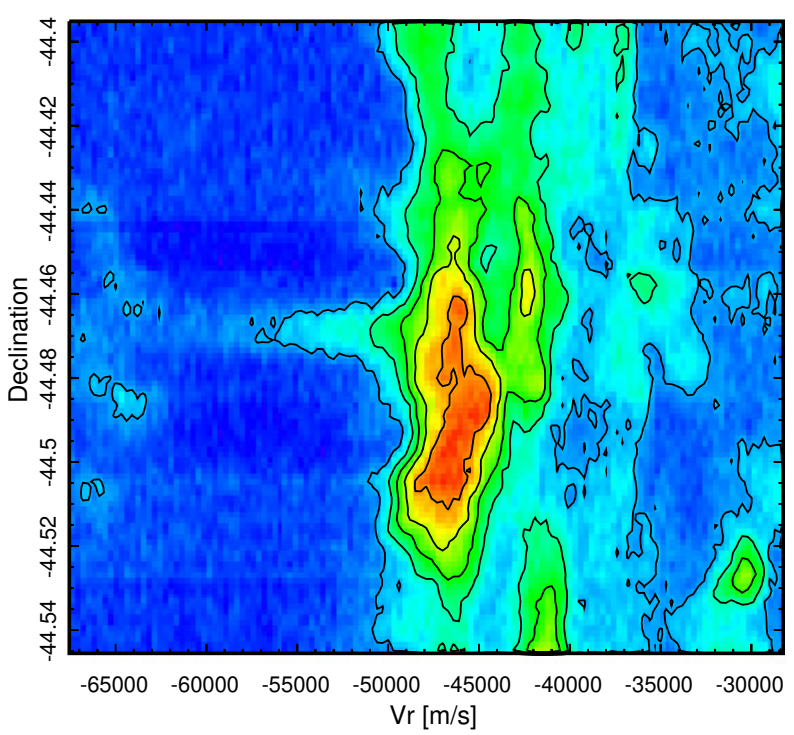

Fig. 11. Position-velocity diagram toward EGO 4 in ${ }^{12} \mathrm{CO}(2-1)$ (Dec. vs. velocity for a fixed value of R.A. $=16: 52: 35)$. The color scale indicates $T_{m b}$ and the declination is indicated in fractions of degrees. The color figure can be viewed online.

establishes, a ratio $M_{\mathrm{vir}} / M\left(\mathrm{H}_{2}\right) \lesssim 1$ indicates that a clump has too much kinetic energy and is unstable against gravitational collapse. Then, the derived ratios suggest that Clump $A$ and Clump $B$ are unstable and could be collapsing.

For the sake of completeness, we calculate the eventual infall rate estimated from Klaassen \& Wilson (2007)

$$
\dot{M}_{\mathrm{inf}}=\frac{4}{3} \pi n_{H 2} \mu m_{H} r_{\text {clump }}^{2} \mathrm{~V}_{\mathrm{inf}},
$$

where $n_{\mathrm{H} 2}$ is the $\mathrm{H}_{2}$ volume density, $r_{\text {clump }}$ is the linear radius of the infall region, and $v_{\text {inf }}$ is the infall velocity of the material. One way to estimate the infall velocity is considering the two layer radiative transfer model of Myers et al. (1996). From the ${ }^{12} \mathrm{CO}(2-1)$ and $\mathrm{C}^{18} \mathrm{O}(2-1)$ spectra corresponding to Clump $A$ in Figure 3, we calculated $\mathrm{v}_{\text {inf }}=0.53 \mathrm{~km} \mathrm{~s}^{-1}$ using equation (9) in Myers et al. (1996). In order to get a better estimate, we also calculated the infall velocity using the Hill5 model (De Vries \& Myers 2005) in the PySpecKit package (Ginsburg \& Mirocha 2011) ${ }^{12}$. The Hill5 model employs an excitation temperature profile increasing linearly toward the center, rather than the two-slab model of Myers et al. (1996), so

\footnotetext{
${ }^{12}$ https://pyspeckit.readthedocs.io/en/latest/ hill5infall_model.html
}

the Hill5 model is thought to provide a better fit to infall motions (De Vries \& Myers 2005). From this model we obtained $\mathrm{v}_{\text {inf }}=1.53 \pm 0.26 \mathrm{~km} \mathrm{~s}^{-1}$. Considering this value of $\mathrm{v}_{\mathrm{inf}}=$ and the parameters listed in Table 3, we obtained $\dot{M}_{\mathrm{inf}} \approx 7.47 \times 10^{-4} M_{\odot} \mathrm{yr}^{-1}$. This result is consistent with those derived from surveys of massive YSOs such as Klaassen \& Wilson (2007). Clump B does not show clear observational evidences of collapse.

A typical outflow appears as spatially confined wings beyond the emission from the cloud core. For EGO 3 and EGO 4, we can distinguish the presence of ${ }^{12} \mathrm{CO}(2-1)$ blue wings from $\approx-60$ to $-50 \mathrm{~km} \mathrm{~s}^{-1}$, shown in the spectra fit in Figure 10, while the velocity range of the optically thin $\mathrm{C}^{18} \mathrm{O}(2-1)$ emission is $\approx-47$ to $-40 \mathrm{~km} \mathrm{~s}^{-1}$. The dashed line marks the systemic velocity of the clumps, which coincides with the central velocity of the $\mathrm{C}^{18} \mathrm{O}(2-1)$ line. Red wings cannot be identified, although multiple components in the ${ }^{12} \mathrm{CO}(2-1)$ emission are present.

The typical signature of outflows can be seen in the position-velocity diagram. As shown in Figure 11 for EGO 4, the extension of the emission toward velocities of about $-55 \mathrm{~km} \mathrm{~s}^{-1}$ would correspond to the blue wings shown in Figure 10. Note that the declination of this extension and the right ascension of the map coincide with the position of EGO 4.

These characteristics suggest that, although they are not spatially resolved, there may be outflows associated with EGO 3 and EGO 4.

\section{SUMMARY}

Based on ${ }^{12} \mathrm{CO}(2-1),{ }^{13} \mathrm{CO}(2-1)$, and $\mathrm{C}^{18} \mathrm{O}(2-1)$ images obtained using the APEX telescope and high density molecular tracers from the MALT90 survey such as $\mathrm{HCO}^{+}(1-0), \mathrm{HNC}(1-0)$ and $\mathrm{N}_{2} \mathrm{H}^{+}(1-0)$ we investigate the molecular component of the IRDC SDC 341.232-0.268. The ${ }^{13} \mathrm{CO}(2-1)$ and $\mathrm{C}^{18} \mathrm{O}(2-1)$ along with MALT90 data reveal two molecular clumps (Clump A and ClumpB) linked to SDC 341.232-0.268 with systemic velocities of $-44.0 \mathrm{~km} \mathrm{~s}^{-1}$, indicating a kinematical distance of $3.6 \pm 0.5 \mathrm{kpc}$. Four EGOs (EGO 1 at RA,Dec $=$ $16: 52: 30.3, \quad-44: 28: 40.0 ; \quad$ EGO 2 at RA,Dec $=$ 16:52:32.2, $-44: 28: 38.0 ; \quad$ EGO 3 at RA,Dec $=$ $16: 52: 34.2,-44: 28: 36.0$ and EGO 4 at RA,Dec $=$ 16:52:37.3, -44:28:09.9) coincide with the molecular clumps. We calculate masses of $2400 \pm 960$ and $1200 \pm 480 M_{\odot}$, for Clump $A$ and Clump B, respectively, and $\mathrm{H}_{2}$ ambient densities $>10^{4} \mathrm{~cm}^{-3}$. Ambient densities estimated using the $\mathrm{N}_{2} \mathrm{H}^{+}(1-0)$ line are higher $\left(\approx 10^{5} \mathrm{~cm}^{-3}\right)$ and agree with the critical 
density of this molecule, which would be tracing the densest part of the clumps.

Both Clump A and Clump B are detected in the FIR (Herschel images) at $\lambda>160 \mu \mathrm{m}$. In the NIR and MIR, at $\lambda<160 \mu \mathrm{m}(24$ and $70 \mu \mathrm{m})$, three out of four EGOs seem to be resolved mainly at $24 \mu \mathrm{m}$. Molecular masses derived from the emission at $870 \mu \mathrm{m}$ are roughly in agreement with those calculated from the molecular lines.

Spectral energy distributions (SEDs) for Clump A and Clump B built using fluxes in the FIR indicate dust temperatures of $13.5 \mathrm{~K}$, typical for an IRDC.

Our search for additional signs of star formation indicates that some of the EGOs coincides with young stellar objects classified as Class I detected as point sources in the Spitzer and WISE catalogs.

Additionally, the ${ }^{12} \mathrm{CO}(2-1)$ spectra toward EGO 3 and EGO 4 present a double-peak structure with the blue-shifted peak brighter than the redshifted one, and with the maximum in the ${ }^{13} \mathrm{CO}(2-1)$ and $\mathrm{C}^{18} \mathrm{O}(2-1)$ spectra coincident with the absorption dip, which reveals the existence of infall material. This fact together with the values obtained from $M_{\text {vir }} / M\left(\mathrm{H}_{2}\right)$ ratio derived for the clumps reveal that they would be collapsing. Blue extended wings in the $\mathrm{C}^{18} \mathrm{O}(2-1)$ spectra are also present toward these EGOs, suggesting the presence of outflows.

This project was partially financed by CONICET of Argentina under projects PIP 00356, and PIP 00107 and from UNLP, projects PPID092, PPID/G002, and 11/G139. M.R. wishes to acknowledge support from CONICYT (CHILE) through FONDECYT grant No1140839. VF acknowledges support from CONICYT Astronomy Program-2015 Research Fellow GEMINI-CONICYT (32RF0002). VF also acknowledges support from the Faculty of the European Space Astronomy Centre (ESAC), and would like to thank Ivan Valtchanov, Bruno Altieri, and Luca Conversi for their support and valuable assistance in Herschel data processing. We thank the referee for the careful reading of the manuscript and constructive comments which greately improved this presentation. This work is based [in part] on observations made with the Spitzer Space Telescope, which was operated by the Jet Propulsion Laboratory, California Institute of Technology under a contract with NASA.

\section{REFERENCES}

Allen, C. W. 1973, Astrophysical quantities, (London: UK: University of London, Athlone Press)
Battersby, C., Bally, J., Jackson, J. M., et al. 2010, ApJ, 721, 222

Benjamin, R. A., Churchwell, E., Babler, B. L., et al. 2003, PASP, 115, 953

Bergin, E. A. \& Tafalla, M. 2007, ARA\&A, 45, 339

Beuther, H., Schilke, P., Gueth, F., et al. 2002, A\&A, 387,931

Brand, J. \& Blitz, L. 1993, A\&A, 275, 67

Breen, S. L., Ellingsen, S. P., Contreras, Y., et al. 2013, MNRAS, 435, 524

Bronfman, L., Nyman, L. A., \& May, J. 1996, A\&ASS, 115,81

Burton, M., Walsh, A., \& Balasubramanyam, R. 2002, ASPC, 267, 355

Cappa, C. E., Duronea, N., Firpo, V., et al. 2016, A\&A, 585, A 30

Carey, S. J., Noriega-Crespo, A., Price, S. D., et al. 2005, AAS, 207, 6333

Caselli, P., Myers, P. C., \& Thaddeus, P. 1995, ApJ, 455, L77

Caswell, J. L., Fuller, G. A., Green, J. A., et al. 2010, MNRAS, 404, 1029

Chambers, E. T., Jackson, J. M., Rathborne, J. M., \& Simon, R. 2009, ApJS, 181, 360

Chen, X., Ellingsen, S. P., Shen, Z.-Q., Titmarsh, A., \& Gan, C.-G. 2011, ApJS, 196, 9

Chen, X., Gan, C.-G., Ellingsen, S. P., et al. 2013, ApJS, 206, 22

Chen, X., Shen, Z.-Q., Li, J.-J., Xu, Y., \& He, J.-H. 2010, ApJ, 710, 150

Contreras, Y., Schuller, F., Urquhart, J. S., et al. 2013, A\&A, 549, A45

Cyganowski, C. J., Whitney, B. A., Holden, E., et al. 2008, AJ, 136, 2391

De Vries, C. H. \& Myers, P. C. 2005, ApJ, 620, 800

Deharveng, L., Zavagno, A., Anderson, L. D., et al. 2012, A\&A, 546, A74

Dickman, R. L. 1978, ApJS, 37, 407

Ellingsen, S. P. 2006, ApJ, 638, 241

Foster, J. B., Jackson, J. M., Barnes, P. J., et al. 2011, ApJS, 197, 25

Foster, J. B., Rathborne, J. M., Sanhueza, P., et al. 2013, PASA, 30, 38

Fuller, G. A., Williams, S. J., \& Sridharan, T. K. 2005, A\&A, 442, 949

Ginsburg, A. \& Mirocha, J. 2011, PySpecKit: Python Spectroscopic Toolkit

Griffin, M. J., Abergel, A., Abreu, A., et al. 2010, A\&A, 518, L3

Guzmán, A. E., Sanhueza, P., Contreras, Y., et al. 2015, ApJ, 815, 130

Hou, L. G. \& Han, J. L. 2014, A\&A, 569, A125

Jackson, J. M., Chambers, E. T., Rathborne, J. M., Simon, R., \& Zhang, Q. 2008, ASPC, 387, 44

Jackson, J. M., Rathborne, J. M., Foster, J. B., et al. 2013, PASA, 30, 057

Klaassen, P. D. \& Wilson, C. D. 2007, ApJ, 663, 1092 
Ladd, N., Purcell, C., Wong, T., \& Robertson, S. 2005, PASA, 22, 62

MacLaren, I., Richardson, K. M., \& Wolfendale, A. W. 1988, ApJ, 333, 821

Mardones, D., Myers, P. C., Tafalla, M., et al. 1997, ApJ, 489, 719

Molinari, S., Swinyard, B., Bally, J., et al. 2010, PASP, 122,314

Myers, P. C., Mardones, D., Tafalla, M., Williams, J. P., \& Wilner, D. J. 1996, ApJ, 465, L133

Offner, S. S. R., Robitaille, T. P., Hansen, C. E., McKee, C. F., \& Klein, R. I. 2012, ApJ, 753, 98

Ohishi, M., Irvine, W. M., \& Kaifu, N. 1992, IAUS, 150, 171

Ossenkopf, V. \& Henning, T. 1994, A\&A, 291, 943

Ott, S. 2010, ASPC, 434, 139

Peretto, N. \& Fuller, G. A. 2009, A\&A, 505, 405

Poglitsch, A., Waelkens, C., Geis, N., et al. 2010, A\&A, 518, L2

Purcell, C. R., Longmore, S. N., Burton, M. G., et al. 2009, MNRAS, 394, 323

Purcell, C. R., Longmore, S. N., Walsh, A. J., et al. 2012, MNRAS, 426, 1972

Rathborne, J. M., Jackson, J. M., \& Simon, R. 2006, ApJ, 641, 389

Rathborne, J. M., Jackson, J. M., Zhang, Q., \& Simon, R. 2008, ApJ, 689, 1141

Rathborne, J. M., Simon, R., \& Jackson, J. M. 2007, ApJ, 662, 1082

Rathborne, J. M., Whitaker, J. S., Jackson, J. M., et al.
2016, PASA, 33, 030

Rawlings, J. M. C., Redman, M. P., Keto, E., \& Williams, D. A. 2004, MNRAS, 351, 1054

Ren, J. Z., Liu, T., Wu, Y., \& Li, L. 2011, MNRAS, 415, L49

Robitaille, T. P. 2008, ASPC, 387, 290

Robitaille, T. P., Whitney, B. A., Indebetouw, R., \& Wood, K. 2007, ApJS, 169, 328

Rohlfs, K. \& Wilson, T. L. 2004, Tools of radio astronomy (Berlin: Springer)

Sanhueza, P., Garay, G., Bronfman, L., et al. 2010, ApJ, 715,18

Sanhueza, P., Jackson, J. M., Foster, J. B., et al. 2012, ApJ, 756, 60

Schuller, F., Menten, K. M., Contreras, Y., et al. 2009, A\&A, 504, 415

Scoville, N. Z., Sargent, A. I., Sanders, D. B., et al. 1986, ApJ, 303, 416

Sobolev, A. M., Ostrovskii, A. B., Kirsanova, M. S., et al. 2005, IAUS, 227, 174

Vassilev, V., Meledin, D., Lapkin, I., et al. 2008, A\&A, 490, 1157

Wang, J., Zhang, Q., Pillai, T., Wyrowski, F., \& Wu, Y. 2008, ApJ, 672, 33

Wright, E. L., Eisenhardt, P. R. M., Mainzer, A. K., et al. 2010, AJ, 140, 1868

Yang, W., Xu, Y., Chen, X., et al. 2017, ApJS, 231, 20

Zhang, Q., Sridharan, T. K., Hunter, T. R., et al. 2007, A\&A, 470, 269

Zhou, S. 1992, ApJ, 394, 204

C. E. Cappa: Facultad de Ciencias Astronómicas y Geofísicas, Universidad Nacional de la Plata, Paseo del Bosque s/n, 1900, La Plata, Argentina (ccappa@fcaglp.unlp.edu.ar).

N. U. Duronea and M. M. Vazzano: Instituto Argentino de Radioastronomía, CONICET, CCT La Plata, C.C.5, 1894, Villa Elisa, Argentina (duronea@iar.unlp.edu.ar, mvazzano@iar.unlp.edu.ar).

V. Firpo: Gemini Observatory, Southern Operations Center, C/o AURA, Casilla 603, La Serena, Chile (vero.firpo@gmail.com).

V. Firpo: Departamento de Astronomía, Universidad de Chile, Casilla 36, Santiago de Chile, Chile (vero.firpo@gmail.com).

C. H. López Caraballo: Departamento de Matemáticas, Universidad de La Serena, Av. Juan Cisternas 1200, La Serena, Chile (lopezcaraballoch@gmail.com).

M. Rubio: Departamento de Astronomía, Universidad de Chile, Casilla 36, Santiago de Chile, Chile (mrubio@das.uchile.cl). 